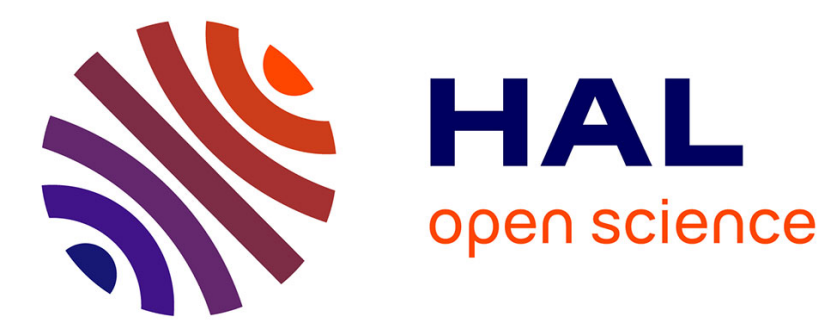

\title{
Towards a comprehensive impossibility result for string stability
}

\author{
Arash Farnam, Alain Sarlette
}

\section{To cite this version:}

Arash Farnam, Alain Sarlette. Towards a comprehensive impossibility result for string stability. IEEE Transactions on Automatic Control, 2019, 10.1109/TAC.2019.2929967 . hal-02394703

\section{HAL Id: hal-02394703 \\ https://hal.inria.fr/hal-02394703}

Submitted on 4 Dec 2019

HAL is a multi-disciplinary open access archive for the deposit and dissemination of scientific research documents, whether they are published or not. The documents may come from teaching and research institutions in France or abroad, or from public or private research centers.
L'archive ouverte pluridisciplinaire HAL, est destinée au dépôt et à la diffusion de documents scientifiques de niveau recherche, publiés ou non, émanant des établissements d'enseignement et de recherche français ou étrangers, des laboratoires publics ou privés. 


\title{
Towards a comprehensive impossibility result for string stability
}

\author{
Arash Farnam, Alain Sarlette
}

\begin{abstract}
We provide a comprehensive impossibility result towards achieving string stability, i.e. keeping local relative errors in check with local controllers independently of the size of a chain of subsystems. We significantly extend existing results, from the LTI setting to any homogeneous controllers that can be nonlinear, time-varying, and locally communicating. We prove this impossibility for a set of definitions with various norm choices, including the $L_{2}$-type standard and a BIBO type criterion. All results hold for a general discrete-time controller which should cover most applications.
\end{abstract}

\section{INTRODUCTION}

String stability roughly requires that a chain of subsystems (e.g. vehicles), controlled by local feedback from relative position measurements and subject to bounded local perturbations, keeps local deformations bounded independently of the size of the chain. In absence of further variables intervening in the system, local deformations are defined as deviations of the distances between consecutive vehicles from a constant target value. The topic has gathered attention from the observation that, for second-order integrator subsystems, using any LTI controller reacting to their predecessor's distance, the amplification of some disturbance components along the chain is unavoidable [1], [2]. The historical application is a chain of acceleration-controlled vehicles, but other distributed systems like mechanical structures [3], [4], or more fundamental models, should also benefit from string stability insight. This has initiated a rich line of results, among others:

- Establishing various impossibility results and scalings for LTI controllers [1], [5], [6], mostly with subsystems reacting to their immediate predecessor and immediate follower. Often PD controllers are used as a proxy for bandwidth limitation, but problems associated to integral control have also been characterized [7].

- Designing a passive LTI controller, with symmetric coupling to predecessor and follower, to keep in check at least in some sense the effect of disturbances acting on the leading subsystem [3].

- Adding a dependence on absolute velocity into the pure double integrator, to overcome the string instability issue [8]-[10]. This can take the form of strong enough drag, which e.g. for terrestrial vehicle applications are easy to ensure but would be in tradeoff with fuel efficiency; in other applications, ensuring strong drag may not always be trivial. Alternatively, the "time-headway"

A.F. and A.S. are with the Department of Electronics and Information Systems (ELIS), Ghent University. Technologiepark 914, 9052 Zwijnaarde, Belgium.

A.S. is with the QUANTIC lab, INRIA Paris. 2 rue Simone Iff, 75012 Paris, France (email: alain.sarlette@inria.fr) spacing policy makes the target inter-vehicle distance dependent on absolute velocity. For terrestrial vehicles again this appears easy to achieve, but questionable in terms of chain performance (possibly big spacing at high velocities, instead of moving efficiently like a big rigid body at all speeds); for other applications, the necessity to measure absolute velocity of each subsystem with respect to a common reference may even pose sensing questions.

- Adding local communication between subsystems, as in adaptive cruise control [11], [12]. Remarkably, existing studies with this approach all incorporate as well a dependence on absolute velocity, as mentioned in the previous item. In the recent paper [13], a variation on time headway is proposed for a generic controlled system and in the nominal case the controller uses both absolute state information and communication with the leader.

We can further refer the reader to the recent review [14]. Research has also been carried out on properties related to, yet different from string stability. Without being comprehensive, we can mention the scaling of linear network eigenvalues with network size [15], the poor robustness of large linear networks under distributed sensing [16], and the coherence of linear networks in presence of stochastic noise [17].

This literature leaves several questions open. How much does each added element (dependence on absolute velocity, local communication capabilities, coupling symmetrically or asymmetrically to one or several vehicles ahead and/or behind) actually contribute towards solving the string stability issue? How much can be gained with more involved, e.g. nonlinear controller designs? What is possible with digital, quantized controllers and realistic modern communication? How much is the LTI setting actually limiting?

The objective of the present note is to answer these questions towards a more precise understanding of string stability and related issues. As a small variation, we consider a discrete-time controller, which is closer to digital controller implementations and incorporates related "natural" constraints in a direct way; although probably most current controllers are digital and discrete-time anyways, the conclusions should carry over in practice to the continuous-time setting as we discuss below. We work only with relative state measurements between neighboring subsystems, e.g. we do not allow controller dependence on absolute velocity. In accordance with the existing literature, we consider an idealized model where input disturbances must be countered while assuming perfect measurements and communication. Our main result is:

We establish that enabling nonlinear controllers, any couplings to a few vehicles in front and behind, any 
(e.g. nonlinear, quantized, event-based) local communication, and controller dependence on the chain length, all together do not allow to design a controller which would achieve string stability with respect to bounded disturbances acting on the subsystems of the chain. We prove this for several variants of the string stability measure, and with as only main constraints: (i) the controller uses relative state measurements between neighboring vehicles; (ii) the controller is homogeneous, i.e. each vehicle (except boundaries) reacts in the same way to its neighbors; (iii) controller discretization step $\Delta t$ remains bounded away from zero despite increasing chain length. The proof comes down to identifying a general counterexample, then working out rather basic computations; we hence believe that the contribution mainly rests on the unprecedented generality of the conclusions. Essentially: string instability in a chain of second-order integrators is an unavoidable property of distributed sensing, for a (much) larger class of controllers than LTI.

The note is organized as follows. Section II describes the setting and several precise definitions of string stability. Section III gives our main result, about a (very) general impossibility of string stability; it ends with a short discussion of discrete-time vs. continuous-time controllers, and an illustrative simulation of the system behavior under a "bad" disturbance. Section IV concludes the note with an outlook on remaining open points and possible further implications.

\section{PROBLEM DEFINITION}

\section{A. Model setting}

We consider a chain of undamped second-order integrators, called "vehicles" for concreteness but which may represent any other (typically mechanical) subsystems coupled with local interactions. In the literature, this continuous-time system

$$
\ddot{x}_{k}(t)=u_{k}(t)
$$

with $x_{k}$ the position and $u_{k}$ the input signal of subsystem $k$ at time $t$, is the standard starting point for string (in)stability [1] [6], [14], with continuous-time e.g. LTI controllers defining the $u_{k}$. We here consider its (exact) equivalent under discrete-time control. We assume that each subsystem is controlled with a discrete-time control logic, computing at each time $t=n \Delta t$ with $n \in \mathbb{Z}$, a control signal that will be applied during the whole interval $(t, t+\Delta t]$ as input to each double integrator; thus $\Delta t$ is the time increment of the discrete-time controller. We can then integrate exactly the second-order dynamics over the time interval $(t, t+\Delta t]$ to obtain:

$$
\begin{aligned}
& v_{k}(t+\Delta t)=v_{k}(t)+u_{k, 1}(t)+d_{k, 1}(t) \\
& x_{k}(t+\Delta t)=x_{k}(t)+v_{k}(t) \Delta t+u_{k, 2}(t)+d_{k, 2}(t),
\end{aligned}
$$

where $x_{k}, v_{k} \in \mathbb{R}$ for $k=0,1,2, \ldots, N$ denote position and velocity respectively; the control inputs $u_{k, 1}, u_{k, 2}$ result from integrating the control signal applied during the interval $(t, t+\Delta t]$ respectively once and twice; and the disturbances $d_{k, 1}, d_{k, 2}$ result from similarly integrating the continuoustime input disturbances. Model (1) is an exact discrete-time equivalent because $u_{k}(t)$ over the interval $(t, t+\Delta t]$ does not depend on the state values after time $t$.

A key constraint is that the feedback signals $u_{k, 1}, u_{k, 2}$ applied between $t$ and $t+\Delta t$ must be based on only relative displacement measurements $e_{k}=x_{k-1}-x_{k}$ between consecutive vehicles in the chain, including possibly relative speeds $\dot{e}_{k}=v_{k-1}-v_{k}$, taken at time $t$. We then consider the following general controller, where $e_{k: \ell}$ denotes for $\ell>k$ the set of values $e_{k}, e_{k+1}, \ldots, e_{\ell}$ :

$$
\begin{aligned}
& u_{k, 1}= f_{1}\left(e_{\left(k-m_{1}\right):\left(k+m_{2}\right)}, \dot{e}_{\left(k-m_{1}\right):\left(k+m_{2}\right)},\right. \\
&\left.c_{k,+}, c_{k,-}, \xi_{k}, N, t\right), f_{2}\left(e_{\left(k-m_{1}\right):\left(k+m_{2}\right)}, \dot{e}_{\left(k-m_{1}\right):\left(k+m_{2}\right)},\right. \\
&\left.c_{k,+}, c_{k,-}, \xi_{k}, N, t\right), \\
& c_{k+1,+}= g_{1}\left(e_{\left(k-m_{1}\right):\left(k+m_{2}\right)}, \dot{e}_{\left(k-m_{1}\right):\left(k+m_{2}\right)},\right. \\
&\left.c_{k,+}, c_{k,-}, \xi_{k}, N, t\right), \\
& c_{k-1,-}= g_{2}\left(e_{\left(k-m_{1}\right):\left(k+m_{2}\right)}, \dot{e}_{\left(k-m_{1}\right):\left(k+m_{2}\right)},\right. \\
& \xi_{k}(t+\Delta t)= h\left(\xi_{k,+}(t), c_{k,-}, \xi_{k}, N, t\right), \\
&\left.\dot{e}_{\left(k-m_{1}\right):\left(k+m_{2}\right):\left(k+m_{2}\right)}, c_{k,+}, c_{k,-}, N, t\right) .
\end{aligned}
$$

Here $m_{1}$ (resp. $m_{2}$ ) is a finite number of agents ahead (resp. behind); $c_{k,+}, c_{k,-} \in \mathbb{R}^{n_{c}}$ with $n_{c}$ some bounded integer are communication signals from $\left\{k-m_{1}, \ldots, k-1\right\}$ to $k$ and from $\left\{k+1, \ldots, k+m_{2}\right\}$ to $k$ respectively; the $\xi_{k} \in \mathbb{R}^{n_{\xi}}$ for some finite integer $n_{\xi}$ allow for dynamical controllers with finite memory; and $f_{1}, f_{2}, g_{1}, g_{2}, h$ are arbitrary functions, with minimal regularity just to ensure that the solution to the dynamical system is well-defined at all times. In particular, by specifying a particular profile of $u(\tau)$ over the discretization time span $\tau \in[t, t+\Delta t)$ between two measurement updates, one can command $f_{1}=\int_{t}^{t+\Delta t} u(\tau) d \tau$ and $f_{2}=\iint_{t}^{t+\Delta t} u(\tau) d \tau^{2}$ independently. The controllers (2) are applied by all vehicles $k \in\left(m_{1}, N-m_{2}\right)$, whereas adapted versions are applied by the $m_{1}$ leading and $m_{2}$ last vehicles. The adapted versions will play no role in the proof.

Note that $m_{1}$ and $m_{2}$ are just opportunities for a vehicle to take into account what happens several vehicles ahead and/or behind. The setting includes unidirectional chains $m_{2}=0$, where vehicles only get inputs from predecessors. It also includes the bidirectional symmetric chain, like encountered in homogeneous mechanical coupling [3], [4], by imposing front-to-back symmetry on (2).

Our aim here is to leave as much freedom as possible on controller design. In particular, the functions $f_{1}, f_{2}, g_{1}, g_{2}, h$ can be nonlinear and time-dependent (e.g. modulated at specific frequencies), thereby vastly extending the traditional LTI setting. Our only true restrictions on control design are:

\section{Assumptions implied by the model:}

- The controllers must be homogeneous along the chain, i.e. the functions $f_{1}, f_{2}, g_{1}, g_{2}, h$ do not depend on vehicle index $k$ and the internal variables are initialized with the same default values for each $k$.

- The digital controller has a finite update time $\Delta t$, fixed independently of chain length $N$. 
- The control commands are based on relative state information only, i.e. there is no dependence on absolute states that would be obtained with respect to some common reference, like absolute velocity.

The first point can be relaxed to approximately homogeneous in our proof; future work, if deemed relevant, could address the fully heterogeneous setting e.g. with adversarial methods. The second point is discussed after the main result. Regarding the third point, we recall that the case with control using absolute velocity has been solved [8], [9]. It is not clear however whether relying solely on absolute velocity for achieving string stability is acceptable in all practical contexts. Without any absolute information is also the original academic question.

\section{B. Control objective}

The goal is to achieve string stability. Roughly said, this means avoiding that the performance gets unboundedly worse when the chain length $N$ grows. Formally, several definitions have been proposed [1]-[3], [6], [7], [12], [14]. Their common point is to focus on stabilizing the $e_{k}$, i.e. the distances between consecutive vehicles. This makes sense e.g. for collision-avoidance, for maintaining a tight platoon, or to avoid too extreme accelerations on the last vehicles of the chain, and is a weaker requirement than controlling the position of every vehicle with respect to the leader. Definitions vary in the norms used to consider the distribution over vehicles, the norm over time of the signals, and the type of disturbance namely as signals or as initial conditions [12], [14]. We here translate the definitions in presence of input disturbances to discrete-time control.

Definition 1: For positive integers $p, q$, the $\ell_{p, q}$ string stability requires that there exist $C_{1}, C_{2}>0$ independent of $N$ such that: for any disturbances satisfying

$$
\sum_{s=1,2} \sum_{k=0}^{N}\left(\sum_{n \in \mathbb{Z}}\left|d_{k, s}(n \Delta t) \frac{1}{\Delta t^{s}}\right|^{p} \Delta t\right)^{q / p}<C_{1},
$$

it is ensured that $\sum_{n \in \mathbb{Z}}\left|e_{j}(n \Delta t)\right|^{p} \Delta t<C_{2}$ for each $j$.

Definition 2: For positive integers $p, q$, the $\left(\ell_{p}, \ell_{q}\right)$ string stability requires that there exist $C_{1}, C_{2}>0$ independent of $N$ such that: for any disturbances satisfying

$$
\sum_{s=1,2} \sum_{k=0}^{N}\left(\sum_{n \in \mathbb{Z}}\left|d_{k, s}(n \Delta t) \frac{1}{\Delta t^{s}}\right|^{p} \Delta t\right)^{q / p}<C_{1},
$$

it is ensured that $\sum_{k=0}^{N}\left(\sum_{n \in \mathbb{Z}}\left|e_{k}(n \Delta t)\right|^{p} \Delta t\right)^{q / p}<C_{2}$.

Definition 3: For positive integer $p$, the $\left(\ell_{p}, \ell_{\infty}\right)$ string stability requires that there exist $C_{1}, C_{2}>0$ independent of $N$ such that: for any disturbances satisfying

$\sum_{n \in \mathbb{Z}}\left|d_{k, s}(n \Delta t) \frac{1}{\Delta t^{s}}\right|^{p} \Delta t<C_{1}, \quad s=1,2$ for all $k$, it is ensured that $\sum_{n \in \mathbb{Z}}\left|e_{j}(n \Delta t)\right|^{p} \Delta t<C_{2}$ for all $j$.

Definition 4: The $\left(\ell_{\infty}, \ell_{\infty}\right)$ string stability requires that there exist $C_{1}, C_{2}>0$ independent of $N$ such that: for any disturbances satisfying $\left|d_{k, s}(t)\right| / \Delta t^{s}<C_{1}$ for $s=1,2$ and all $k, t$, it is ensured that $\left|e_{j}(t)\right|<C_{2}$ for all $j, t$.

The $\Delta t$ factors are introduced because $d_{k, 1}$ and $d_{k, 2}$ are supposed to result from integrating (respectively once and twice) a continuous-time signal $d_{k}(t)$ over the time interval $(t, t+\Delta t]$. In principle we could just choose units such that $\Delta t=1$, but we keep $\Delta t$ for later discussion. The main point about string stability is to satisfy the constraints with constants independent of the number of vehicles $N$.

Definition 1 is the weakest since it imposes a bound on the vector norm of disturbance inputs, but in return it only requires each individual $e_{j}$ to have a bounded signal norm. It is a necessary condition for achieving stronger versions of string stability, and it has been considered a lot in the literature; e.g. until recently this was the only proven working definition for a controller depending on absolute velocity, as we exclude here [8]-[10]. The other definitions each consider the same norm on input disturbances and on output errors, either summing them over vehicles (Def.2) or not (Def.3, Def.4). The most popular norm has been $p=q=2$, especially the time-integration with a 2-norm has attracted a lot of attention thanks to its equivalent formulation in frequency domain. Definition 4 changes the treatment of time to formulate a BIBO type version of string stability. It seems to have attracted little attention in the literature, probably because most of the literature has focused on LTI frequency domain approaches. However, we would argue that this version is closest to practical concerns; and, as we will show that the string instability issue is not limited to the LTI setting, we will use tools that can treat this definition explicitly too. In the particular context of (1),(2), or when restricting the controllers, it might well be that some of the above definitions hold strictly together; in absence of further evidence, we will treat them all.

Remark 1: The definitions were initially stated in the linear context, where $C_{1}$ and $C_{2}$ can be rescaled such that it makes no difference in which order they are chosen (e.g. variants like "for each $C_{1}$, there exists a $C_{2}$ " become equivalent to our statement). In the nonlinear context this might differ, and we have chosen the weaker constraint: thus our impossibility results will also hold for stronger variants.

Remark 2: The disturbance model appears quite standard: each subsystem is subject to some disturbance input, bounded in some sense, and the consequences on the system must be bounded. A possible point of discussion though is that this standard definition of string stability checks system behavior under the worst disturbance satisfying the constraints. If the worst disturbance can get rejected, then we have a strong guarantee, and this is the typical approach for studying system stability. However, if the behavior under the worst disturbance does not behave well as $N$ gets large, it might also be that this worst disturbance becomes increasingly unlikely among all possible combinations of disturbance signals. In other words, in a probabilistic model of all possible input disturbance signals, it may be that the probability to have a problematic input signal converges to 0 as $N$ increases to infinity. More positive results have indeed been obtained with such a probabilistic model, although not for a chain of subsytems, see e.g. [17]. We here stick to the standard definition, thus considering the worst bounded input signal. 


\section{MAin IMPOSSIBILITY ReSUlT}

We now prove that Definitions 1-4 are all impossible to satisfy even with a general controller as allowed by (2). The main idea of the proof is to construct a disturbance input which is badly countered by any distributed controller. We take advantage of a simple construction that focuses on exactly solving the central part of the chain only, in order to give a lower bound on the induced error.

A simulation illustrating the behavior of the system under this construction can be found in Section III-D.

\section{A. A badly countered disturbance situation}

Consider disturbances of the following form:

$$
\begin{aligned}
d_{k, 1}(t)=d_{k, 2}(t)=0 & \text { for all } t<0, k=0,1, \ldots, N \\
d_{k, 1}(t)=\frac{\alpha k \Delta t}{N}, & \text { for all } t=0, \Delta t, \ldots, T, \\
\left.d_{k, 2}(t)=\frac{\alpha k \Delta t^{2}}{N}\right\} & k=0,1, \ldots, N ; \\
d_{k, 1}(t)=d_{k, 2}(t)=0 & \text { for all } t>T, k=0,1, \ldots, N,
\end{aligned}
$$

with constants $\alpha>0$ and $T>0$ to be specified later.

To compute the evolution of the system under these disturbances, we exploit the finite propagation speed of signals along the chain - namely at most $\left(m_{1}, m_{2}\right)$ vehicles per time step - to restrict our attention to a central subset of vehicles.

- Consider the evolution of $e_{k}$ and $\dot{e}_{k}$ over one time step, when the $N+1$ vehicles all start with the same state $x_{k}(0)=$ $v_{k}(0)=0$ for all $k$, and with controllers initialized at $\xi_{k}=$ $c_{k,+}=c_{k,-}=0$ for all $k$. We get

$$
\begin{aligned}
e_{k}(\Delta t)= & e_{k}(0)+\Delta t \dot{e}_{k}(0)+u_{k-1,2}(0)-u_{k, 2}(0) \\
& \quad+d_{k-1,2}(0)-d_{k, 2}(0) \\
= & u_{k-1,2}(0)-u_{k, 2}(0)+\alpha \Delta t^{2} / N \\
\dot{e}_{k}(\Delta t)= & \dot{e}_{k}(0)+u_{k-1,1}(0)-u_{k, 1}(0) \\
& \quad+d_{k-1,1}(0)-d_{k, 1}(0) \\
= & u_{k-1,1}(0)-u_{k, 1}(0)+\alpha \Delta t / N
\end{aligned}
$$

Since the $e_{k}(0)$ and $\dot{e}_{k}(0)$ are all equal, it is clear that the control inputs are all equal too, i.e. $u_{k-1,1}=u_{k, 1}$ and $u_{k-1,2}=u_{k, 2}$, at least for all vehicles with $m_{1}<k<N-m_{2}$. Completely irrespectively of the controller chosen, we thus have for all $m_{1}<k<N-m_{2}$ :

$$
e_{k}(\Delta t)=\alpha \Delta t^{2} / N \quad \text { and } \quad \dot{e}_{k}(\Delta t)=\alpha \Delta t / N .
$$

Also the $c_{k,+}(\Delta t), c_{k,-}(\Delta t)$ and $\xi_{k}(\Delta t)$ will be equal.

- Now consider a time $t=n \Delta t$ for some integer $n>0$ and assume that all the state variables satisfy equalities $e_{k}(t)=$ $e_{j}(t), \dot{e}_{k}(t)=\dot{e}_{j}(t), c_{k,+}(t)=c_{j,+}(t), c_{k,-}(t)=c_{j,-}(t)$ and $\xi_{k}(t)=\xi_{j}(t)$ for all $j, k \in\left[N_{\text {lead }}, N-N_{\text {tail }}\right]$, for some integers $N_{\text {lead }}, N_{\text {tail }}>0$. Slightly extending the above example, we get:

$$
\begin{array}{r}
e_{k}(t+\Delta t)=e_{k}(t)+\Delta t \dot{e}_{k}(t)+u_{k-1,2}(t)-u_{k, 2}(t) \\
+d_{k-1,2}(t)-d_{k, 2}(t) \\
=e_{k}(t)+\Delta t \dot{e}_{k}(t)+\alpha \Delta t^{2} / N=e_{j}(t+\Delta t) \\
\text { for all } j, k \in\left[N_{\text {lead }}+m_{1}, N-\left(N_{\text {tail }}+m_{2}\right)\right] \\
\dot{e}_{k}(t+\Delta t)=\dot{e}_{k}(t)+\alpha \Delta t / N=\dot{e}_{j}(t+\Delta t)
\end{array}
$$$$
\text { for all } j, k \in\left[N_{\text {lead }}+m_{1}, N-\left(N_{\text {tail }}+m_{2}\right)\right] \text {, }
$$

and similarly we maintain $c_{k,+}(t)=c_{j,+}(t), c_{k,-}(t)=c_{j,-}(t)$ and $\xi_{k}(t)=\xi_{j}(t)$ for that subset of vehicles.

By iterating this argument we get the following property.

Lemma 1: Consider the system (1),(2) subject to the particular disturbance (3) and zero initial conditions. Then for any (well-defined) controller, the solution satisfies:

$$
\begin{aligned}
& e_{k}(t)=t(t+\Delta t) \alpha /(2 N) \\
& \dot{e}_{k}(t)=t \alpha / N,
\end{aligned}
$$

for all $t \in[0, T]$ and all $k \in\left(\frac{t}{\Delta t} m_{1}, N-\frac{t}{\Delta t} m_{2}\right)$.

Proof: The main argument is provided by the explanations preceding the statement. From (4), the $\dot{e}_{k}$ is obtained as a sum of $t / \Delta t$ times the bias $\alpha \Delta t / N$. Then replacing this into the expression of $e_{k}$ in (4), one observes that the increment of $e_{k}$ at time $n=t / \Delta t$ is linear in $n$, so the standard formula for a linearly progressing series gives the result.

To be useful at time $t$, the solution (5) of Lemma 1 should cover at least 1 vehicle, i.e. $N-\frac{t}{\Delta t}\left(m_{1}+m_{2}\right) \geq 1$. For fixed $m_{1}, m_{2}$ and $\Delta t$, we can ensure to have a valid solution for at least $N / 2$ vehicles over the interval $[0, T]$, by taking $T=\frac{N \Delta t}{2\left(m_{1}+m_{2}\right)}$. As $m_{1}$ and $m_{2}$ are constants independent of $N$, we essentially suggest to select the duration $T$ of the "bad" input disturbance to be of order $N \Delta t$.

\section{B. Consequences for string stability}

We now investigate what the above construction implies for string stability. First take Definition 1. For the proposed disturbance, the condition on $d_{k, 1}, d_{k, 2}$ becomes:

$$
2 T^{q / p}\left(\frac{\alpha}{N}\right)^{q} \sum_{k=0}^{N} k^{q}<C_{1} .
$$

For large $N$, the dominating term in the sum is $N^{q+1}$ such that we need in fact $2 T^{q / p} \alpha^{q} N<C_{1}$ or in other words, $\alpha$ of order $1 /\left(N^{1 / q} T^{1 / p}\right)$. This fixes the allowed disturbance amplitude as a function of its duration and of $N$.

For the vehicles covered by Lemma 1, we then have

$$
\begin{aligned}
\sum_{n \in \mathbb{Z}}\left|e_{j}(n \Delta t)\right|^{p} \Delta t & \geq \sum_{n=0}^{T / \Delta t}\left|e_{j}(n \Delta t)\right|^{p} \Delta t \\
& \simeq\left(\frac{\alpha}{2 N}\right)^{p} \sum_{n=0}^{T / \Delta t}(n \Delta t)^{2 p} \Delta t \\
& \simeq\left(\frac{\alpha}{2 N}\right)^{p} \Delta t^{2 p+1}\left(\frac{T}{\Delta t}\right)^{2 p+1} .
\end{aligned}
$$

Towards the last line we have again used the dominating term in the sum. Combining this with the just obtained bound on $\alpha$ and with taking $T$ of order $N \Delta t$ as suggested at the end of Section III-A, we obtain that $\sum_{n \in \mathbb{Z}}\left|e_{j}(n \Delta t)\right|^{p}$ is at least of order $N^{p-p / q} \Delta t^{2 p}$.

A similar argument can be repeated for the other definitions of Section II, yielding the following results.

Theorem 2: For the system (1),(2), there exist disturbances $d_{k, 1}$ and $d_{k, 2}$ satisfying the required respective bounds according to the definitions of Section II and such that, irrespectively 
of any (well-defined) controller choice, for large $N$ :

[Definition 1]: $\sum_{n}\left|e_{j}(n \Delta t)\right|^{p}$ grows as $N^{p-p / q} \Delta t^{2 p}$;

[Definition 2]: $\sum_{k=0}^{N}\left(\sum_{n}\left|e_{k}(n \Delta t)\right|^{p}\right)^{q / p}$ grows as $N^{q} \Delta t^{2 q}$;

[Definition 3]: $\sum_{n}\left|e_{j}(n d t)\right|^{p}$ grows as $N^{p} \Delta t^{2 p}$;

[Definition 4]: $\left|e_{j}(t)\right|$ grows as $N \Delta t^{2}$.

Proof: We will always assume $T$ of order $N \Delta t$ and consider the output errors for $t \in[0, T]$. The computation for Def. 1 is given above. For Def. 2 it is the same, but taking the power $q / p$ and summing the disturbance over the number of vehicles for which Lemma 1 is valid - this can be of order $N$ as mentioned in the last paragraph of Section III-A. For Def.3, the disturbance can be larger i.e. $\alpha$ of order $1 / T^{1 / p}$, and with respect to the Def.1 computation this adds a factor $N^{p / q}$ to the output error. For Def.4, we can have $\alpha$ of order 1 , and since the result of Lemma 1 is valid for $T$ of order $N \Delta t$ we can have $e_{k}(t)$ of order $T(T+1) \alpha / N \sim N^{2} \Delta t^{2} / N$.

For $\Delta t$ fixed and $N$ growing to infinity, this result establishes impossibility to satisfy any of the definitions of string stability given in Section II, except possibly Def.1 with $q=1$ (which does not appear to have any practical significance, see comments about the Definitions in Section II). This impossibility is established in a very general setting, allowing unidirectional or bidirectional symmetric or asymmetric coupling, looking a number of vehicles ahead and behind (as long as that number is independent of $N$ ), communicating with neighbors with any encoding/decoding schemes with possibly packets and event-based logic, and processing all this in an arbitrary nonlinear control system with memory. In particular, even perfect local communication among the vehicles is not sufficient, on its own, to ensure string stability. It is thus no wonder that vehicle chain controllers with realistic communication channels have so far required an additional feedback from absolute velocity to achieve string stability [11]-[13].

\section{How telling is the discrete-time controller setting?}

The reader will have noticed that the above impossibility breaks down if we let $\Delta t$ converge to zero fast enough as $N$ grows to infinity. While this does not look like a practical solution, it may express a relevant tradeoff; and, it does create a gap with the pure continuous-time literature. We will thus briefly comment on the comparison of this result with the literature on continuous-time, typically LTI systems.

First, note that since our result only follows a sufficient construction, Theorem 2 proves that it is necessary - yet possibly not even sufficient - to let $\Delta t$ go to zero with increasing $N$ in order to satisfy string stability. And indeed, for e.g. PD coupling with nearest neighbors, we know from LTI results that our disturbance would yield string instability in continuous-time too. This may not be too surprising as a lowfrequency disturbance appears to cause most of the problem. Our argument leading to Thm.2 thus indeed appears to be quite too optimistic still. Now let us try to discuss which controller features would typically go with a very small $\Delta t$ :
- One obvious effect of smaller $\Delta t$ is faster communication across the vehicle chain. If one could communicate arbitrarily fast, perfectly and without measurement errors, then each vehicle $k$ could get very fast knowledge of $e_{1}+e_{2}+\ldots+e_{k}=x_{k}-x_{0}$. One can then obviously achieve string stability: just control each $x_{k}-x_{0}$ independently to stabilize each vehicle with respect to the leader. The "distributed system" setting and chain size $N$ play no role anymore. Of course this idealized situation is unrealistic. In reality, precision of a message (and in fact of a measurement) is in a clear tradeoff to update speed. As long as the communication bandwidth per signal remains bounded when $N$ increases, the imperfections resulting from smaller $\Delta t$ are likely to counterbalance the apparent benefits of smaller $\Delta t$ from our perfectcommunication model.

- Setting sensing and communication aside, in practice, the controller's discretization step $\Delta t$ is chosen as the desired dwell-time before vehicle $k$ reacts to a measurement; thus in practice $\Delta t$ converging to zero would mean, controller bandwidth tending to infinity, pointing towards controllers with gain increasing as a function of $N$. It is known indeed that academically speaking, this can provide string stability: in continuous-time, without communication, a LTI controller whose gain increases fast enough with $N$, can ensure string stability. However, as the control gain keeps increasing towards infinity, effects of unmodeled system limitations and imperfections cannot be neglected forever and practical problems are likely to appear.

- Theorem 2 thus shows anyways that string stability is, at best, not robust to time-discretization. This is important to know towards system simulations, where situations that work only for infinitesimal $\Delta t$ are quickly considered non-robust for all practical purposes. In a sense, testing robustness to finite $\Delta t$ can even be mathematically compared to the traditional requirement of "no poles cancellation" in the continuous-time setting. Indeed, allowing a decreasingly small $\Delta t$ without any measurement noises can be compared to allowing precise computation of $\lim _{\Delta t \rightarrow 0} \frac{s(t+\Delta t)-s(t)}{\Delta t}$ for a signal $s$, i.e. evaluating pure derivatives. For a double-integrator, this implies the possibility of pole cancellation at zero frequency, which is almost always excluded.

- The dependence on $\Delta t$ is rooted in the fact that we analyze the system before the signals from the edges of the chain reach all the vehicles and make a detailed analysis harder. This does not mean of course that the vehicle chain would automatically be stabilized as soon as the signals from the edges have crossed the chain, see e.g. the simulations in Section III-D. In this sense, it appears that the boundary controllers would play a key role towards string stabilizing the system with infinitesimal $\Delta t$, similarly to PDE control.

These arguments give strong indications to conjecture that string stability would be impossible with any "reasonable" homogeneous, possibly nonlinear, and communicating controllers, in continuous-time too. At this point of detail, we 
might argue as well that the digital-controller model is in fact closer to applications, than the traditional continuous-time one.

Remark 3: To further connect this result to existing work, we can look at how the chain reacts to disturbances acting on the first subsystem only. This has indeed been considered in several continuous-time LTI studies, which we first review now. For unidirectional coupling, reaction to leader-disturbances is sometimes viewed as a major indicator of general behavior [1], [2]. For bidirectional chains under symmetric coupling, although impossibility results are known from e.g. [7], under the condition of disturbance restricted to the leading vehicle, $\ell_{2,2}$ string stability has been established in [3]. Pushing further the idea of [15] and of [18], [19] about possible advantages of slight mistuning in the controller symmetry, we have proved in [20] that a sufficiently asymmetric bidirectional PD controller is sufficient to ensure also the stronger versions of string stability, with respect to disturbances restricted to a fixed number of leading vehicles. The proof uses an analytic almost-inversion of the system equations based on forward and backward flows, loosely inspired from [21].

This line of work can be related in two ways to the result of the present paper. First, we have checked that a discrete-time version of our string stability result in [21] can be worked out perfectly well for the model (1),(2), see [22]. This suggests that our discrete-time model does enable positive results when the continuous-time model does, i.e. it adds evidence in favor of (1),(2) not being essentially more constraining than the more standard continuous-time approach. Second, the disturbance proposed in Section III-A is extensively distributed along the vehicle chain, as a function of $N$. This is consistent with a picture of two regimes: when disturbances act on a few vehicles (at known places!), it may be possible to reject them in a string stable way; however when they are distributed along the whole chain, there is no way to achieve string stability on the basis of relative measurements only.

\section{Illustrative simulation}

We can of course only illustrate the string instability for a particular choice of controller. However, trusting in the simple analysis of Section III-A, our main argument is independent of controller choice. Thus, we will just show how indeed the solution of Lemma 1 appears for a simple linear controller without communication. We choose this simplicity to avoid selecting too many elements in the controller design "arbitrarily" since according to Theorem 2, any attempt is anyways doomed to fail. Complementarily the simulation shows what happens once the "boundary effects" have propagated throughout the chain i.e. when the solution of Lemma 1 is not valid anymore; this will depend on the choice of controller, but it falls outside the scope of the present paper. We thus suggest that the reader should not draw too strong conclusions from what happens outside the scope of Lemma 1 with this particular controller.

We take a hint from [18], [19] and select a PD controller having bidirectional coupling, with gain on position feedback symmetric towards the preceding and following vehicle, but gain on velocity asymmetric. Considering a simple sampleand-hold digital actuation, we will thus assume that $u_{k}(\tau)=$

$$
\begin{aligned}
f(t, k):= & b_{1}\left(v_{k-1}(t)-v_{k}(t)\right)+b_{2}\left(v_{k+1}(t)-v_{k}(t)\right) \\
& +a\left(x_{k-1}(t)-x_{k}(t)\right)+a\left(x_{k+1}(t)-x_{k}(t)\right)
\end{aligned}
$$

for all $\tau \in(t, t+\Delta t]$ in continuous-time. After exact integration this yields the exact discrete-time model (1) with

$$
u_{k, 1}(t)=f(t, k) \Delta t, \quad u_{k, 2}(t)=f(t, k) \Delta t^{2} / 2 .
$$

The simulation takes arbitrary values $a=1, b_{1}=2, b_{2}=0.5$, and $\Delta t=0.1$; for the first and last vehicle, we just drop from the feedback law the term associated to the missing neighbor.

The precise scaling of input disturbances to apply and of output signals to monitor, depends on the definition of string stability that one wishes to consider. We will illustrate the BIBO type scaling of Definition 4, with $\alpha=1$ independent of $N$. As the illustrated controller is linear, it is just a matter of scaling to translate the simulation to other definitions.

To better illustrate the effect of $N$ only, we will make a small variation on the applied disturbance. Indeed, since our analysis in Lemma 1 only goes up to time $t=T$, for a causal system, it does not matter which disturbance we apply for $t>T$. Therefore, we take an input disturbance of the form (3), but instead of stopping it at $t=T$ which scales with $N$, we apply this same nonzero input for all $t>0$, i.e. we are examining a sort of step response. In this way, the input disturbance acting on vehicle say $k=N / 2$ becomes independent of $N$, as illustrated on Fig. 1. The impossibility to achieve BIBO string stability via Lemma 1 will be visible as the fact that, when $N$ grows, the maximum deviation $\left|e_{N / 2}(T)\right|$ at time $T=N \Delta t / 5$ grows unboundedly.

On Figure 2, we show the simulated spacing error between consecutive vehicles under this model, up to $t=T$ and for two different chain lengths $N=10$ and $N=50$. The black squares are the solution given by Lemma 1 . One indeed observes that many (central) vehicles follow this solution, while others are progressively affected by the boundary effects and behave differently. Note that as $N$ increases, in accordance with Lemma 1, the error at a given time becomes lower; this is due to the consecutive vehicles' disturbance inputs becoming more similar as $N$ increases. However, in return, the solution of Lemma 1 remains valid for a longer time $T$ and therefore overall the error at time $T$, that we can easily compute, keeps increasing unboundedly with $N$.

We can also have a look at the behavior of the chain for $t>T$, see Figure 3. A striking observation is that the errors keep increasing way beyond the point covered by our analysis (black dot very close to the origin). However, as this depends on the chosen controller we must be careful about further conclusions. The main conclusion might thus just be that for a fixed $N$, the chosen controller indeed stabilizes the errors to bounded values, i.e. it does effectively stabilize the system. Only, it does not so uniformly in $N$, and this is what string instability essentially means.

\section{CONCLUDING DISCUSSION}

This paper significantly extends the scope of an impossibility result regarding string stability towards input disturbances 

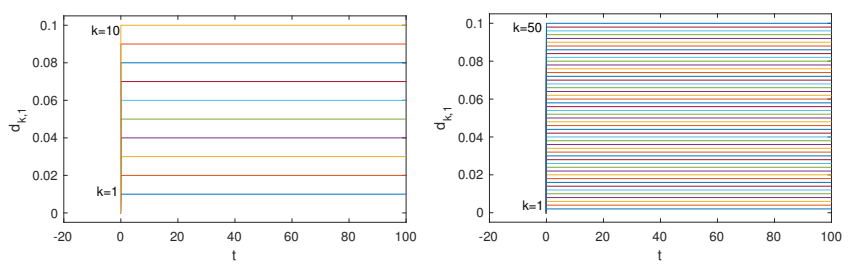

Fig. 1. Disturbance inputs applied to the vehicles, according to (3) but without limiting the time window to $t<T$ (see main text), for $N=10$ and for $N=$ 50 vehicles respectively. The figure is showing $d_{k, 1}(t)$ as the corresponding $d_{k, 2}(t)$ are just the same multiplied by $\Delta t$.
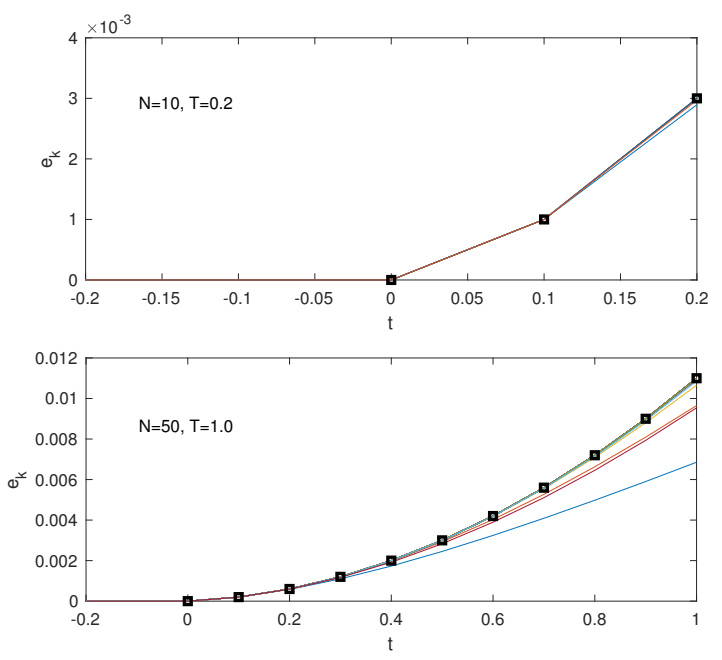

Fig. 2. Evolution of the distance errors $e_{k}(t)$ for $t \geq T$, for a vehicle chain (see main text for details) subject to the input disturbances shown on Fig. 1, and for $N=10$ and $N=50$ respectively. Note the different scales on both axes. In agreement with our analysis we have taken $T=N \Delta t / 5$. For this time interval, a significant number of $e_{k}(t)$ is supposed to follow the solution described by Lemma 1; the latter is plotted as black dots which indeed superimpose with a number of simulated curves.
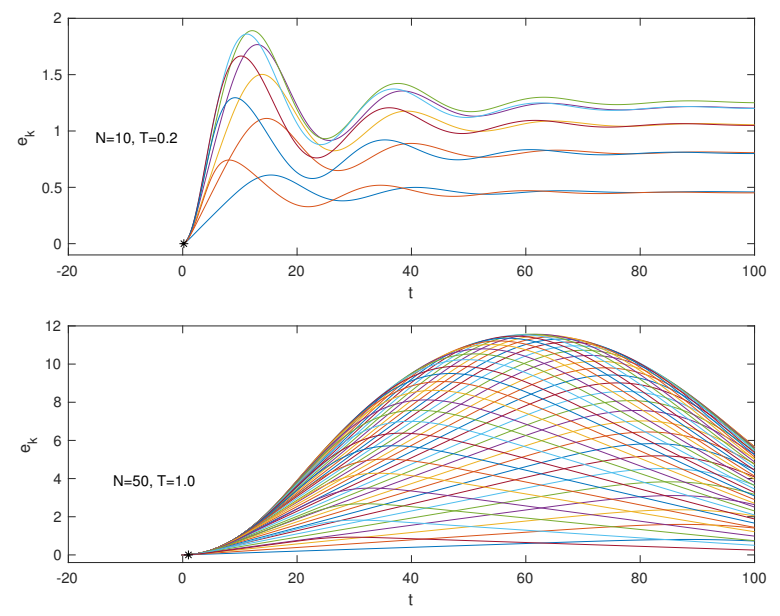

Fig. 3. Evolution of the $e_{k}(t)$ for larger times $t$, i.e. the plots of Figure 2 are in fact zooms on the beginning of the present plots. For $O(t)>T$ (marked with a black dot), the result of Lemma 1 no longer holds and the system behavior does depend on the particular controller choice. We observe that, with the chosen controller: (i) for fixed $N$ indeed each error eventually stabilizes to a bounded value; and (ii) the analysis of Lemma 1, i.e. with errors taken into account only up to time $t=T$, is very optimistic. acting on all subsystems. Indeed, while existing results have centered on LTI systems, we here allow controllers to be nonlinear, $N$-dependent, time-varying, thus possibly modulated and digitally quantized - as well as using any type of perfect local communication at finite speed. The analysis involves no complicated elements once the setting and example are identified, but as the search for alternative controllers had remained open so far, it appears to give a definite answer clearly narrowing down the options towards achieving string stability. Essential features for the impossibility are:

- Second-order integrator model for individual subsystems: if the dynamics was first-order, our counterexample would not work;

- Relative measurements: variations that do solve string stability by adding an absolute velocity term are known, see e.g. time-headway policies [8]-[10]. With respect to this criterion, academically, string instability appears more than ever as a property of distributed sensing. In practice, using absolute velocity in the feedback controller or damping becomes a question of hardware and application tradeoff. We must mention that the string instability issue is not directly linked to the low observability for long-range modes in distributed systems with relative measurements [16]. Indeed, here the target variables are not the absolute displacements $x_{k}$, for which indeed there would be an observability issue, but rather the relative displacements $e_{k}$, which are directly measured. Also see the previous point.

- Homogeneous controller, i.e. same logic with same parameter values at all vehicles: technically, the possibility remains that heterogeneous controllers, i.e. letting the different vehicles react differently to the same signals, could solve the issue. However, we currently have no clue how to design this heterogeneity - unless one would allow parameters increasing unboundedly with chain length $N$, which however would pose other obvious problems. Controllers periodic in vehicle number, do not seem to work.

- Discrete-time controller: this should be representative in practice of a realistic digital controller. Rigorously, our counterexample analysis would break down when reducing the discretization step $\Delta t$ with $N$. However, a property that only holds with infinitely large bandwidth $1 / \Delta t$ for communication and/or control, is usually not robust in practice; this suggests that any "reasonable" continuous-time controller would fail too. Note that the standard string stability model here includes no measurement nor communication imperfections, while with extreme continuous-time controllers that are badly modeled by finite $\Delta t$ those can be expected to become important.

Also note that we have only identified one particular, badly rejected disturbance input. There may be disturbance inputs for which the system reaction is even worse.

With this we believe to have given at least a much more comprehensive picture of what can be done on the standard academic property of string stability. If this string stability 
property appears critical in some key applications, those results should help guide a possible search for very particular controllers to achieve it, if it is feasible at all. A point that we did not study is string stability with respect to disturbances on the initial state, instead of on input signals; a similar analysis might be possible.

A different option for the future is to acknowledge that the academic definition of string stability is too strong to be useful, thus even in an extended framework with nonlinear controllers and so on. In that sense, we can think of two reasonable variations on string stability.

- One option is to consider the tradeoff in a more integrated picture for finite $N$ : to have a given acceptable error, what are the best possible combinations of absolute-velocitydependence $h$, control+communication bandwidths $1 / \Delta t$, possibly nonlinear effects, and associated gains in presence of other noises, as a function of chain length $N$ ? This, knowing that the limit for infinite $N$ will not work, but will also not be essential for most applications.

- Another approach would be, as already mentioned in Remark 2, to acknowledge that the worst-case formulation of string stability is too strong: as the worst-case disturbance could become more and more unlikely with increasing $N$, it might be more telling to take the limit $N \rightarrow \infty$ with a probability distribution over disturbances. In [17], precisely this approach is taken for the behavior of a lattice of simple linearly coupled systems.

As a final word, we may reflect on the more profound implications of our impossibility result. The investigation of [17] for instance is motivated by the stability of physical matter, which after all appears to be governed by forces depending on relative states. Implications are also expected for the numerical simulation of related PDEs. It may be an important theoretical aim to pin down what essential element in the system structure leads to this impossibility.

A first point in this direction is that the analysis towards our Theorem 2 can be easily extended to other spatial interconnection structures, e.g. a $D$-dimensional lattice of $N$ possibly nonlinear systems:

- We can keep our counterexample with $d_{k}$ increasing along one dimension of the lattice from 0 to $\alpha$ with steps $\frac{\alpha}{N^{1 / D}}$, and constant along the other dimensions.

- Computing the acceptable $T$ and $\alpha$ for each case, we get the relevant error growing like $N^{\beta}$ where $\beta \geq 0$ depends on the lattice dimension and on the choice of definition, but it is always $>0$ for Definitions 2-4.

Compared to [17], we thus generalize the setting by allowing any nonlinear, time-varying local interactions towards improving the situation, but we obtain a more negative result by considering the worst bounded disturbance, over time and over subsystem indices. If the aim is to break a system into parts, this particular disturbance may be useful insight. In contrast, to understand the stability of lattices in a natural environment, one may have to acknowledge that bad disturbances in fact become negligibly probable with increasing $N$. This sets a maybe unexpected link between distributed systems and error correcting codes, where scaling to larger codes must essentially rely on the increasing unlikelihood of uncorrectable errors [23].

\section{REFERENCES}

[1] D. Swaroop and J. Hedrick, "String stability of interconnected systems," IEEE Trans. Automatic Control, Volume 41, Pages 349-357, 1996.

[2] D. Swaroop, "String stability of interconnected systems: An application to platooning in automated highway systems," PhD thesis, University of California, Berkeley, 1994.

[3] Y. Yamamoto, and M. C. Smith, "Bounded disturbance amplification for mass chains with passive interconnection," IEEE Trans. Automatic Control, Volume 47, Pages 2534-2542, 2015.

[4] Y. Yamamoto, and M. C. Smith, "Design of passive interconnections in tall buildings subject to earthquake disturbances to suppress inter-storey drifts," Journal of Physics: Conference Series, 2016.

[5] S. Sheikholeslam and C. Desoer, "Longitudinal control of a platoon of vehicles," Proc. American Control Conf., Pages 291-297, 1990.

[6] P. Seiler, A. Pant, and K. Hedrick, "Disturbance propagation in vehicle strings," IEEE Trans. Automatic Control, Volume. 37, Pages 1835-1842, 1996.

[7] P. Barooah and J. P. Hespanha, "Error amplification and disturbance propagation in vehicle strings with decentralized linear control," Proc. IEEE Conf. on Decision and Control, Pages 4964-4969, 2005.

[8] S. Klinge, and R. H. Middleton, "Time headway requirements for string stability of homogenous linear unidirectionally connected systems," Proc. IEEE Conf. on Decision and Control, Pages 1992-1997, 2009.

[9] J.A. Rogge and D. Aeyels, "Vehicle Platoons Through Ring Coupling," IEEE Trans. Automatic Control, Volume 53, Pages 1370-1377, 2008.

[10] S. Knorn, A. Donaire, J.C. Aguero and R.H. Middleton, "Passivitybased control for multi-vehicle systems subject to string constraints," Automatica, Volume 50, Pages 3224-3230, 2014.

[11] J. Ploeg, D.P. Shukla, N. van de Wouw and H. Nijmeijer, "Controller synthesis for string stability of vehicle platoons," IEEE Trans.Intell.Transp. Systems, Volume 15, Pages 854-865, 2014.

[12] J. Ploeg, N. van de Wouw, and H. Nijmeijer, " $L_{p}$ String Stability of Cascaded Systems: Application to Vehicle Platooning," IEEE Trans. Control Systems Technology Volume 22, Pages 786-793, 2014.

[13] B. Besselink and K. H. Johansson, String Stability and a Delay-Based Spacing Policy for Vehicle Platoons Subject to Disturbances, IEEE Trans. Automatic Control, Volume 62, Pages 4376-4391, 2017.

[14] S. Stüdli, M.M. Seron and R.H.Middleton, "From vehicular platoons to general networked systems: String stability and related concepts," Annual Reviews in Control, Volume 44, Pages 157-172, 2017.

[15] P. Barooah, P. G. Mehta and J. P. Hespanha, "Mistuning-based control design to improve closed-loop stability of vehicular platoons," IEEE Trans. Automatic Control, Volume 54, Pages 2100-2113, 2009.

[16] A. Sarlette and R. Sepulchre, "Control limitations from distributed sensing: theory and Extremely Large Telescope application", Automatica, Volume 50, Pages 421-430, 2014.

[17] B. Bamieh, M. R. Jovanovic, P. Mitra, and S. Patterson, "Coherence in Large-Scale Networks: Dimension Dependent Limitations of Local Feedback," IEEE Trans. Automatic Control, Volume 57, Pages 22352249, 2012.

[18] I. Herman, S. Knorn and A. Ahlen, "Disturbance scaling in bidirectional vehicle platoons with different asymmetry in position and velocity coupling," Automatica, Volume 82, Pages 13-20, 2017.

[19] I. Herman, D. Martinec, Z. Hurak and M. Sebek, "Nonzero bound on Fiedler eigenvalue causes exponential growth of $\mathrm{H}$-infinity norm of vehicular platoon," IEEE Transactions on Automatic Control Volume 60, Pages 2248-2253, 2015.

[20] A. Farnam and A. Sarlette, "String Stability towards Leader thanks to Asymmetric Bidirectional Controller," 20th IFAC World Congress, 2017.

[21] W.J. O'Connor, "Wave-based analysis and control of lump-modeled flexible robots," IEEE Trans. Robotics, Volume 23, Pages 342-352, 2007.

[22] A. Farnam, "Towards impossibility and possibility results for string stability of platoon of vehicles," PhD Thesis, Ghent University, 2018.

[23] F.G. MacWilliams and N.J.A. Sloane, The theory of error-correcting codes, Elsevier, 1977. 